\title{
Normothermic Ex Vivo Machine Perfusion for Liver Grafts Recovered from Donors after Circulatory Death: A Systematic Review and Meta-Analysis
}

\author{
Jordan J. Nostedt $\mathbb{D}^{1},{ }^{1}$ Daniel T. Skubleny, ${ }^{1}$ A. M. James Shapiro $\mathbb{D},{ }^{1}$ Sandra Campbell, ${ }^{2}$ \\ Darren H. Freed, ${ }^{3,4,5}$ and David L. Bigam $\mathbb{D}^{1}$ \\ ${ }^{1}$ Department of Surgery, Division of General Surgery, University of Alberta Hospital, 2D4.41 W.M.C, 8440-112 St., \\ Edmonton, AB, Canada T6G $2 B 7$ \\ ${ }^{2}$ John W. Scott Health Sciences Library, University of Alberta, 2K3.28 W.M.C, 8440-112 St., Edmonton, AB, Canada T6G $2 B 7$ \\ ${ }^{3}$ Department of Physiology, University of Alberta, 7-55 Medical Sciences Building, Edmonton, AB, Canada T6G 2H7 \\ ${ }^{4}$ Department of Biomedical Engineering, University of Alberta, 1098 Research Transition Facility, 8308-114 St., Edmonton, AB, \\ Canada T6G 2 V2 \\ ${ }^{5}$ Department of Surgery, Division of Cardiac Surgery, University of Alberta and 4A7.056 Mazankowski Alberta Heart Institute, \\ 11220-83 Ave, Edmonton, AB, Canada T6G 2B7
}

Correspondence should be addressed to David L. Bigam; dbigam@ualberta.ca

Received 15 December 2017; Accepted 15 March 2018; Published 23 April 2018

Academic Editor: Shusen Zheng

Copyright (C) 2018 Jordan J. Nostedt et al. This is an open access article distributed under the Creative Commons Attribution License, which permits unrestricted use, distribution, and reproduction in any medium, provided the original work is properly cited.

\begin{abstract}
As a result of donation after circulatory death liver grafts' poor tolerance to cold storage, there has been increasing research interest in normothermic machine perfusion. This study aims to systematically review the current literature comparing normothermic perfusion to cold storage in donation after circulatory death liver grafts and complete a meta-analysis of published large animal and human studies. A total of nine porcine studies comparing cold storage to normothermic machine perfusion for donation after circulatory death grafts were included for analysis. There was a significant reduction in AST (mean difference $-2291 \mathrm{U} / \mathrm{L}, \mathrm{CI}(-3019$, -1563 ); $P \leq 0.00001$ ) and ALT (mean difference $-175 \mathrm{U} / \mathrm{L}, \mathrm{CI}(-266,-85) ; P=0.0001$ ), for normothermic perfusion relative to static cold storage, with moderate $\left(I^{2}=61 \%\right)$ and high $\left(I^{2}=96 \%\right)$ heterogeneity, respectively. Total bile production was also significantly higher (mean difference $=174 \mathrm{ml}, \mathrm{CI}(155,193) ; P \leq 0.00001)$. Further research focusing on standardization, performance of this technology following periods of cold storage, economic implications, and clinical trial data focused on donation after circulatory death grafts will be helpful to advance this technology toward routine clinical utilization for these grafts.
\end{abstract}

\section{Introduction}

Liver transplant remains the only definitive therapy for end stage liver disease. However the shortage of quality organs remains significant in the United States with 1673 patients dying while on the waitlist and a further 1227 removed, too sick to undergo transplant during 2015 [1]. Due to organ shortage, there has been a rise in the use of extended criteria donors (ECD). These donors include those with significant steatosis, advanced age, and donation after circulatory death (DCD) liver grafts [2].
DCD grafts represent an important source of organs to expand the donor pool. The number of DCD grafts used continues to increase; however there is also a rise in the percentage of DCD grafts recovered but not transplanted [1]. This is a result of these grafts' poor tolerance to static cold storage (SCS) [3], the current standard for organ preservation. DCD grafts are more prone to reperfusion injury and susceptible to ischemic biliary cholangiopathy. As a result, outcomes of DCD transplants have traditionally been marginal showing lower long-term patient and graft survival and increased biliary complications [4]. More recent results show improved 
graft and patient survival, though ischemic cholangiopathy is still a frequent complication of DCD grafts [5].

Ex vivo perfusion is now being studied as a method of increasing use of DCD grafts. Studies using hypothermic and subnormothermic perfusion have shown promising results in both large animal [6-9] and clinical studies [10]; however, in marginal grafts such as those from DCD, normothermic machine perfusion (NMP) showed superior graft function and preservation of biliary epithelium in animal models [11, 12]. In addition to organ preservation, NMP offers the advantage of being able to assess graft viability during perfusion under physiologic conditions where the graft is metabolically active. It also provides opportunity to deliver and monitor response to therapies in order to resuscitate marginal grafts prior to transplantation. These added benefits have led to growing research interest in NMP for DCD grafts in an effort to expand the organ donor pool. NMP for DCD grafts have been studied primarily in large animal studies where resource allocation only allows for small study subject numbers, and study design is critical to advance this complex technology. Although not used often in animal research, systematic reviews can have an important role for the development of future studies [13]. To our knowledge this is the first systematic review of NMP for DCD liver grafts with a meta-analysis of published data.

The aim of this paper is to systematically review the current literature comparing NMP to SCS in DCD liver grafts in large animal (pig) and human studies. The secondary aim is to complete a meta-analysis of NMP versus SCS livers in published DCD porcine liver perfusions.

\section{Methods}

2.1. Search Strategy. Searches were conducted in Ovid MEDLINE, OVID EMBASE, EBSCO CINAHL, WOS, SCOPUS, Proquest Dissertations and Theses, and PROSPERO by an expert librarian (SC) in June 2017 and updated in July 2017. Searches employed both controlled vocabularies (e.g., MeSH, EMTREE) and key words such as (DCD livers) and (ex vivo perfusion or normothermic perfusion). Search strategies were adapted for each database. Search strategies are available in the supporting information (S1). No limits were applied.

All full text, porcine, and human trials comparing NMP to SCS for the preservation of DCD livers were included for analysis. Studies that did not include DCD livers and those that focused only on hypothermic or subnormothermic machine perfusion were excluded.

2.2. Selection of Studies. Titles and abstracts from the primary search were reviewed independently by two authors (JN, DS) for studies that met inclusion criteria. When this was not clear from the titles and abstracts, full text articles were reviewed to determine inclusion.

2.3. Outcome Measures. Primary outcomes in ex vivo perfusion studies included assessment of alanine aminotransferase (ALT) and aspartate aminotransferase (AST) levels as markers of hepatocellular damage, as well as bile production and lactate clearance as markers of liver function. Secondary outcomes were histological preservation and hemodynamic stability indicated by hepatic arterial flow. Primary outcomes in orthotopic pig liver transplant studies included posttransplant peak AST, bile production, and graft survival. Secondary outcomes included histologic preservation. Where there was missing data for quantitative analysis, this information was requested via email from the publication corresponding authors. We received two responses but no further data for inclusion. Where possible, this data was estimated from published figures using Adobe Acrobat Reader DC software.

2.4. Assessment of Bias. Articles were assessed by two authors (JN, DS) using the Systematic Review Centre for Laboratory Animal Experimentation (SYRCLE) risk of bias assessment tool [14].

2.5. Statistical Analysis. A trained statistician performed statistical analysis. Outcomes assessed in the meta-analysis included AST, ALT, total bile production, and hepatic artery flow for perfusion studies, as well as peak AST for transplant studies. They are all continuous variables expressed as mean \pm standard deviation (SD). The mean difference (MD) was used as a summary measure of efficacy between groups treated by NMP and SCS. When no SD was provided, a pooled SD was estimated as previously described [15]. Meta-analysis was performed using RevMan 5.3 software. Heterogeneity of studies was assessed and the following cut-offs were applied, low $(>25 \%)$, moderate $(>50 \%)$, and high $(>75 \%)$ as described by Higgins et al. [16].

\section{Results}

3.1. Search Results. Three hundred and eighty-six titles were identified through our primary search, with 228 remaining for screening after the removal of duplicates. Of these, 201 titles were excluded for the following reasons: published abstract with no complete full text article, comparison of hypothermic or subnormothermic perfusion without NMP, and studies without DCD grafts. Nine articles that directly compared cold storage to NMP for DCD grafts were included for analysis (Figure 1). Six articles were perfusion studies $[11,17-21]$ and two pig transplant models [22, 23]. One article published results of both a perfusion model and pig transplant model [12]. There are no clinical trials directly comparing SCS to NMP specifically for DCD livers and as such the studies included for analysis were limited to porcine experimental studies. The results of included studies are summarized in Tables 1 and 2.

3.2. Pig Liver Ex Vivo Perfusion Studies. Pooled data showed a significant reduction in AST at the end of the simulated transplant phase in the NMP group relative to SCS (MD = $-2291 \mathrm{U} / \mathrm{L}$, CI $(-3019,-1563) ; P \leq 0.00001)$. A similar trend was seen in ALT $(\mathrm{MD}=-175 \mathrm{U} / \mathrm{L}, \mathrm{CI}(-266,-85) ; P=$ $0.0001)$. However the heterogeneity was moderate $\left(I^{2}=61 \%\right)$ and high $\left(I^{2}=90 \%\right)$, respectively, for these two variables (Figure 2). 


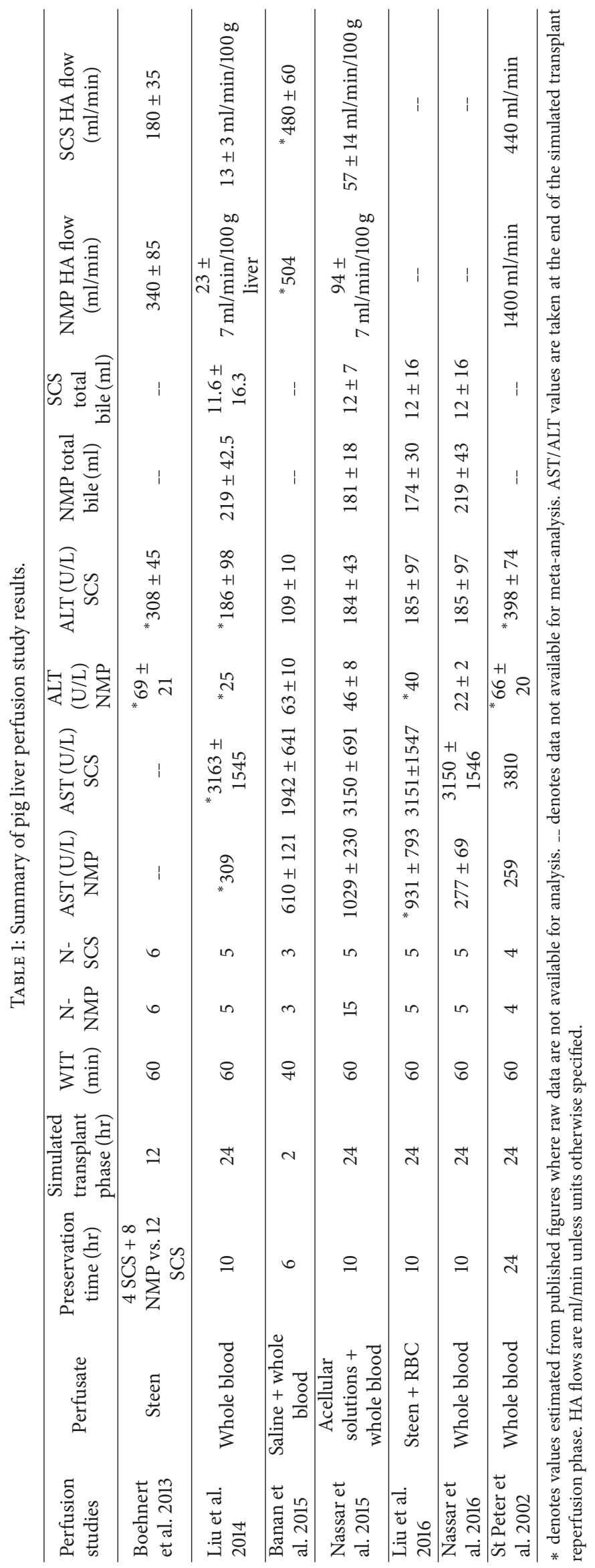


TABLE 2: Summary of pig orthotopic liver transplant studies.

\begin{tabular}{|c|c|c|c|c|c|c|}
\hline $\begin{array}{l}\text { Pig transplant } \\
\text { studies }\end{array}$ & $\begin{array}{l}\text { Preservation } \\
\text { time (hr) }\end{array}$ & $\begin{array}{c}\text { Duration of } \\
\text { posttrans- } \\
\text { plant } \\
\text { monitoring }\end{array}$ & $\mathrm{NMP} n=$ & $\operatorname{SCS} n=$ & $\begin{array}{l}\text { NMP peak } \\
\text { AST (U/L) }\end{array}$ & $\begin{array}{l}\text { SCS peak } \\
\text { AST(U/L) }\end{array}$ \\
\hline $\begin{array}{l}\text { Schön et al. } \\
2001\end{array}$ & 4 & 7 days & 6 & 6 & $603 \pm 141$ & $1570 \pm 171$ \\
\hline $\begin{array}{l}\text { Fondevila et } \\
\text { al. } 2011\end{array}$ & 4 & 5 days & 6 & 6 & $692 \pm 77$ & $1500 \pm 269$ \\
\hline $\begin{array}{l}\text { Boehnert et } \\
\text { al. } 2013\end{array}$ & 12 & 8 hours & 6 & 6 & $524 \pm 187$ & $1809 \pm 205$ \\
\hline
\end{tabular}

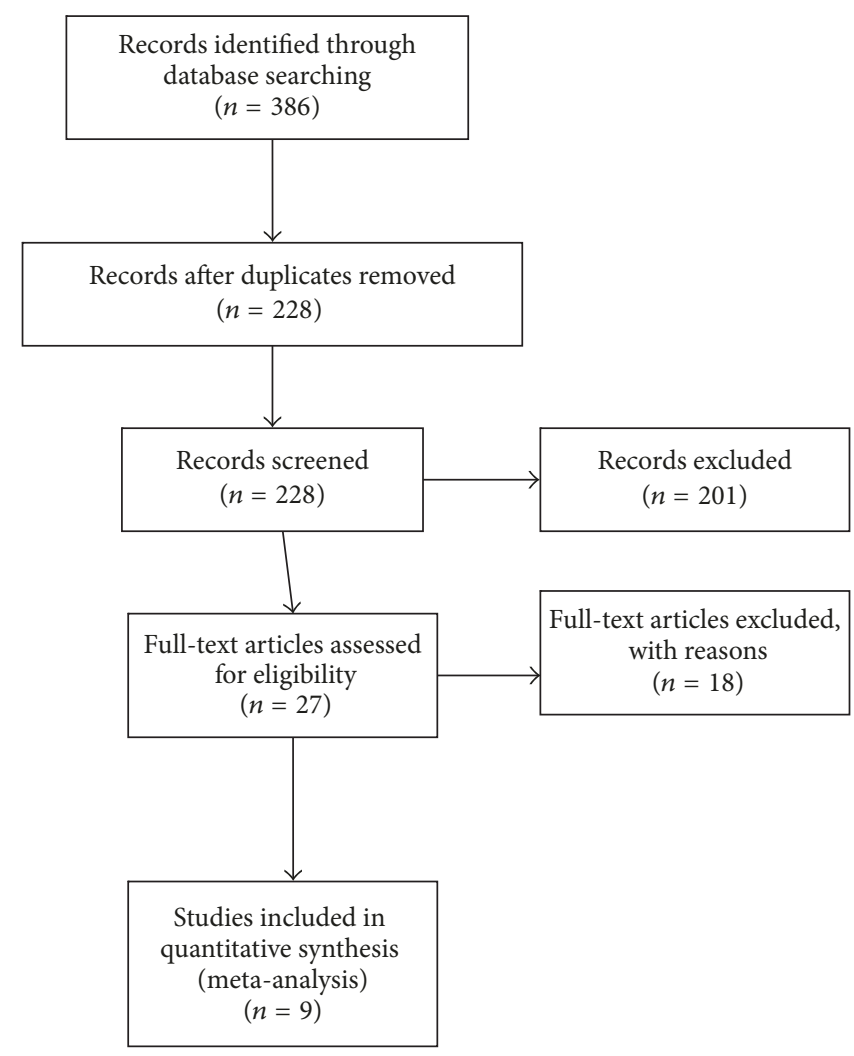

FIGURE 1: Study selection.

Total bile production following the simulated transplant phase was significantly higher in the NMP group (MD $=174 \mathrm{ml}$, CI $(155,193) ; P<0.00001)$. There was low heterogeneity $\left(I^{2}=45 \%\right)$ (Figure 2 ).

There was insufficient data available to perform metaanalysis for lactate clearance.

Limited data was available for hepatic arterial flow. The NMP group did demonstrate higher flows, although this did not reach statistical significance $(P=0.09)$ (Figure 2).

Different histological scoring systems were used by different centers and thus were not suitable for meta-analysis. All perfusion studies showed less necrosis and improved architectural preservation in the NMP group relative to SCS [11, 12, 17-21]. Similarly, NMP demonstrated improved preservation of the biliary epithelium and peribiliary plexus $[11,17,20]$.

3.3. Pig Liver Orthotopic Transplant Studies. Posttransplant peak AST was lower in the NMP group (MD $=-1019$, CI $(-1276,-762) ; P<0.00001)$. There was a high level of heterogeneity $\left(I^{2}=78 \%\right.$ ) (Figure 3$)$. There was insufficient data available to compare bile production. Graft survival also was not assessed in the meta-analysis, as the recovery period in each of these studies was different (Table 2). Boehnert et al. 


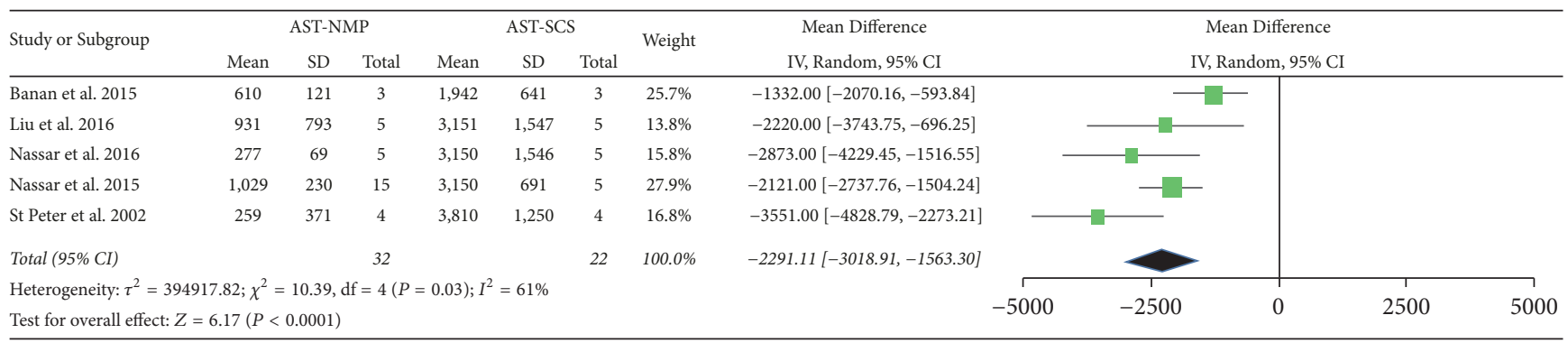

(a)

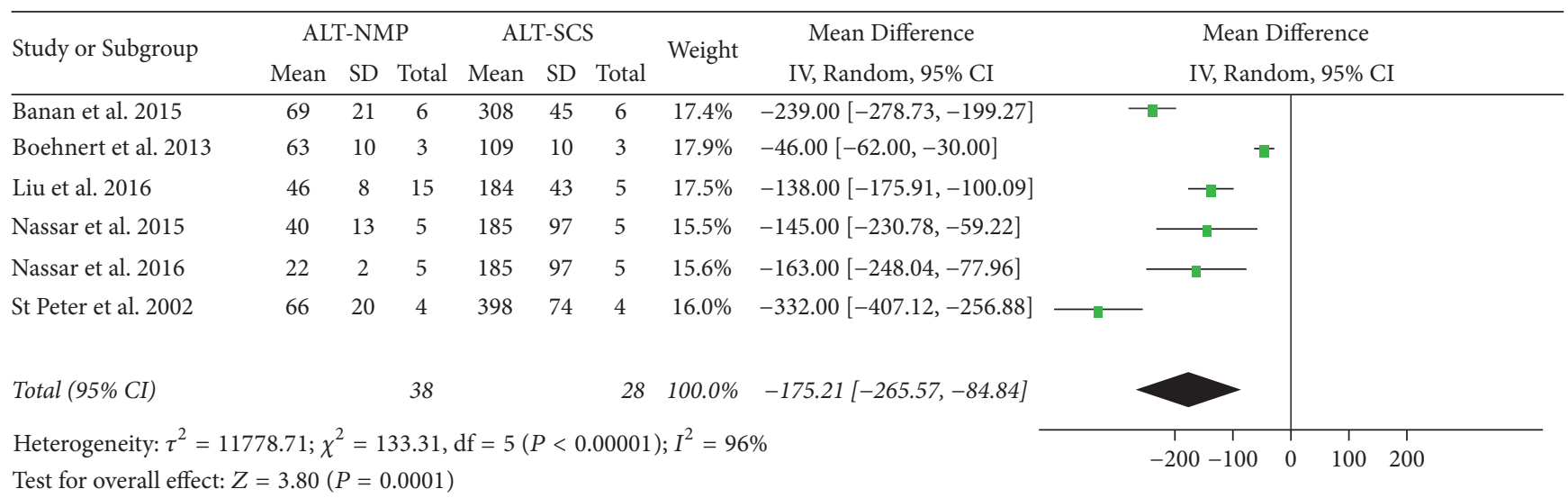

(b)

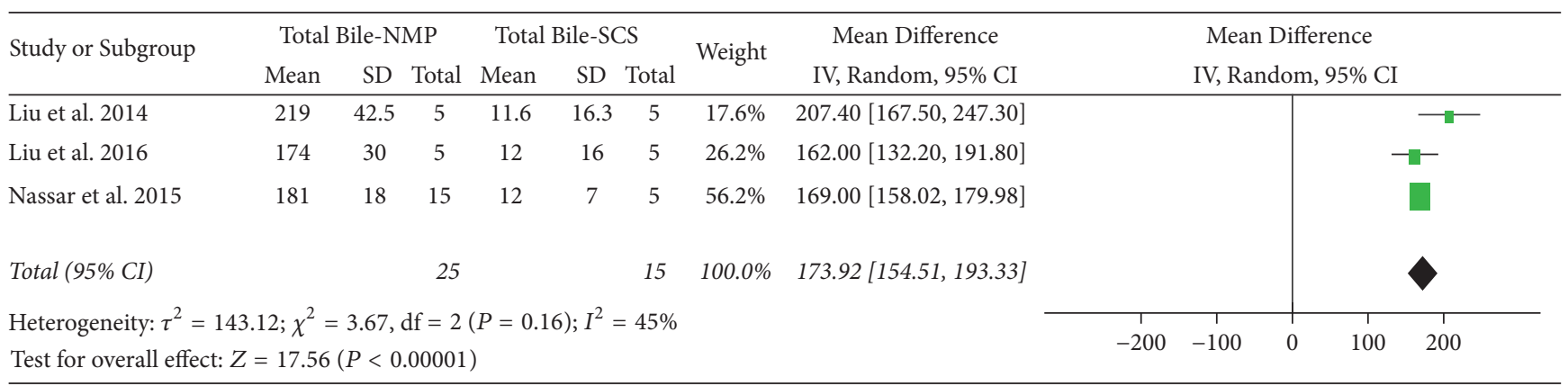

(c)

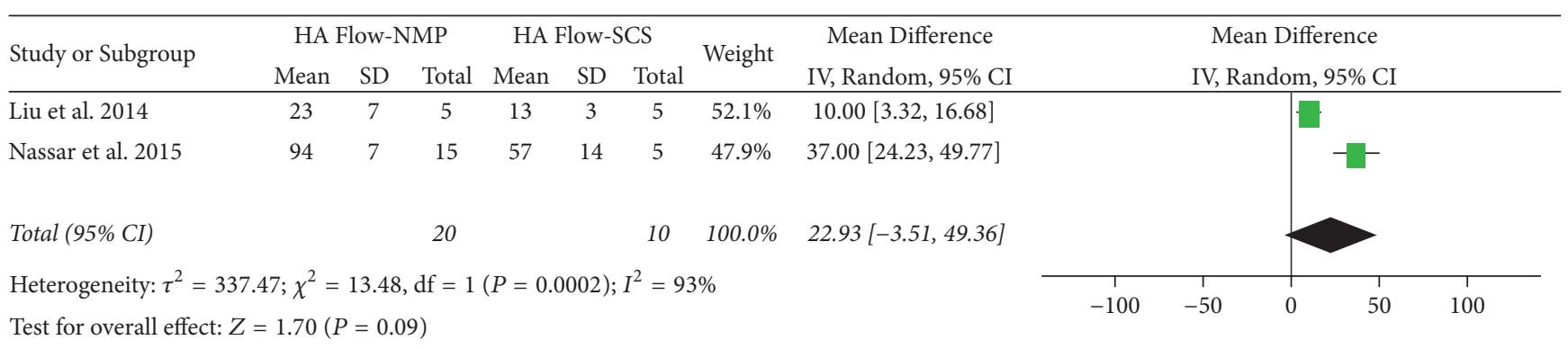

(d)

FIGURE 2: Forest plots showing pooled AST, ALT, and bile production data from porcine liver perfusion studies.

reported no difference in bile production in the eight hours following transplant after perfusing with acellular solution [12]. Schön et al. showed all grafts transplanted after $60 \mathrm{~min}-$ utes of WIT and SCS suffered primary graft nonfunction [22].
In an uncontrolled DCD transplant model where normothermic extracorporeal membrane oxygenation was combined with either NMP or SCS, there was $100 \%$ five-day survival in the NMP group relative to $83 \%$ survival in the SCS group [23]. 


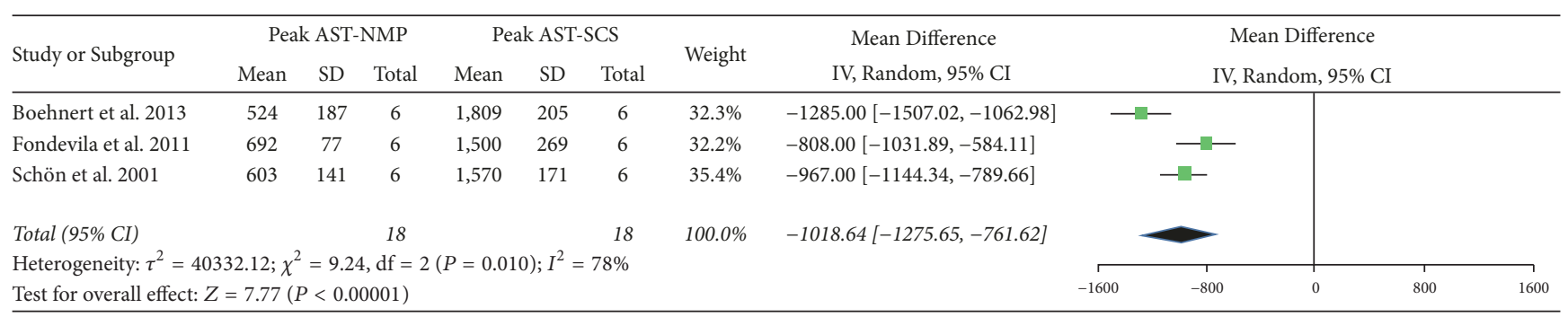

FIGURE 3: Forest plots showing pooled peak AST data from porcine orthotopic liver transplant studies.

NMP groups demonstrated less necrosis, sinusoidal swelling, and improved overall architectural preservation relative to SCS groups $[22,23]$. One pig transplant model did not report histologic data [24].

3.4. Risk of Bias Assessment. The allocation process of animals was unclear in several studies [12, 21-23]; however no other significant sources of bias within the included studies were identified.

\section{Discussion}

The results of this review and meta-analysis must be interpreted with caution, as heterogeneity was high within the perfusion studies limiting the strength of conclusions that can be drawn. Experimental design for the included perfusion studies varied in several fundamental parameters. Major differences included surgical model, duration of preservation and reperfusion, and ex vivo circuit design.

Pigs used as liver donors were $30-40 \mathrm{~kg}$ and included landrace $[18,21]$ and Yorkshire $[11,12,17,19,20]$, with male pigs used only by Boehnert et al. [25] and gender unspecified ones in one study [21]. The DCD model also varied between studies with the majority inducing cardiac arrest with potassium chloride injection [11, 17-21], while one study induced cardiac arrest via exsanguination [12].

Boehnert et al. work was the only perfusion study to compare SCS to NMP following a period of SCS [12]. The WIT in all included perfusion studies was 60 minutes except for Banan et al. [18] who compared SCS after 40 minutes of WIT to NMP following 20, 40, and 60 minutes of WIT. Following WIT livers were flushed with histidine-tryptophanketoglutarate [11,17-20], University of Wisconsin [12], or Euro Collins [21] cold preservation solutions. Livers were flushed in situ $[11,12,18,19,21]$ or ex situ $[17,20]$ with dual perfusion through the hepatic artery and portal vein $[11,17-20]$ or single arterial flush [21]. One study did not specify if dual vessel flush was used [12].

NMP was then carried out for either 6 [18], 8 [12], 10 $[11,17,19,20]$, or 24 [21] hours. Simulated transplant with whole blood reperfusion was for either 2 [18], 12 [12], or 24 $[11,17,19-21]$ hours, which is of important note as transaminase levels were reported at the end of the reperfusion stage.

One study included a dialysis circuit as part of the perfusion setup [18]. Flow was driven by either dual centrifugal pumps [18], the combination of a centrifugal pump and roller pump $[11,17,19,20]$, or a centrifugal pump to perfuse the hepatic artery and the portal vein perfused by gravity [12, 21]. With regard to perfusate used, three studies used whole blood $[11,17,21]$, two used dilute whole blood [18, 19], and one used acellular perfusate [12]. The study by Liu et al. is the only one to directly compare different perfusates [20], using Steen solution, Steen solution with washed red blood cells, and whole blood compared to SCS. Hepatocellular injury and liver function were significantly better in the Steen solution with red blood cells and whole blood groups relative to both SCS or Steen solution alone. There was no significant difference between the whole blood or Steen solution with washed red blood cells [20]. Within the included studies there was not enough available data to perform subgroup analysis based on type of perfusate used. However, the results with acellular perfusion [12] to our knowledge have not been replicated and more studies are still needed to determine the optimal NMP perfusate composition for DCD livers; however the results from Liu et al. [20] suggest the need for an oxygen carrier.

In porcine liver transplant models there was also significant study heterogeneity. The posttransplant observation period ranged from eight hours to seven days. Fondevila et al. [23] compared NMP to SCS following a period of normothermic extracorporeal machine oxygenation, which was significantly different from the other included transplant studies. Schön et al. compared SCS to NMP with no period of SCS and all grafts that were exposed to 4 hours of cold storage following 60 minutes of WIT suffered primary nonfunction [22]. This is in keeping with previous data suggesting that even brief periods of cold storage can impact positive effects of NMP [3]. The study by Boehnert et al., however, compared SCS alone to a period of SCS followed by NMP and reported less hepatocellular injury in the NMP group [12], but data from these grafts were only reported for eight hours after transplant and longer-term survival of the grafts was not assessed. In discarded human liver studies, NMP has shown the ability to recover function of damaged livers even after extensive periods of cold storage [26]. Further research to address NMP's ability to safely recover and transplant DCD grafts following periods of cold storage is needed. Devices available for NMP were reviewed by Ravikumar et al. [27] and portable perfusion devices are now available to try and eliminate cold storage time in the transplant sequence for these marginal organs. Whether NMP can successfully recover DCD grafts after periods of cold storage remains an important question that will impact the clinical implementation of ex vivo NMP. The economic impact of these systems has not yet been studied and will also remain 
a factor in clinical implementation of NMP for DCD grafts. The use of gradual rewarming has shown promise for this population of liver grafts [28-31] and may play an important role moving forward in utilizing machine perfusion after periods of SCS.

NMP has shown capacity to recover function in discarded DCD human liver studies [26, 32-35] and has been used to recover these grafts for clinical transplant $[36,37]$. NMP has also been studied as a method to assess which marginal DCD grafts are safely transplantable. A set of viability criteria has been proposed by Mergental et al. [37]. Establishing a standardized set of criteria will be an important goal for clinical implementation of NMP for DCD grafts.

There are phase I clinical trials comparing NMP to SCS [38-40]; however these studies have only limited numbers of DCD and otherwise marginal grafts. To date no randomized control trials have been published comparing NMP to SCS specifically in DCD grafts. Results of a multicenter European randomized control trial (ISRCTN39731134) comparing NMP to SCS, once published, may be pivotal for this technology moving forward into clinical practice.

\section{Limitations}

There was a large amount of heterogeneity amongst the small number of studies as outlined above. These significant differences in experimental design limit the strength of conclusions that could be drawn from meta-analysis. Furthermore, multiple data points included for meta-analysis were estimated from published figures which may differ slightly from the measured values.

\section{Conclusion}

Meta-analysis of published porcine perfusion studies demonstrates that NMP is superior to SCS regarding the preservation of liver architecture and function in DCD grafts. Given significant differences between studies, these results are to be taken with caution. Further study is still required in order to optimize and standardize perfusate composition and to evaluate NMP's role in preservation following periods of cold storage. Clinical studies involving more DCD grafts will help bring this technology closer to clinical implementation. Economic factors need to be considered in subsequent studies to ensure feasibility within current healthcare systems.

\section{Conflicts of Interest}

The authors declare that there are no conflicts of interest regarding the publication of this article.

\section{Acknowledgments}

The authors would like to thank Chunhong Tian for statistical assistance.

\section{Supplementary Materials}

Supplementary materials include the literature search strategy for this systematic review and meta-analysis (S1). (Supplementary Materials)

\section{References}

[1] W. R. Kim, J. R. Lake, and J. M. Smith, "OPTN/SRTR 2013 annual data report: liver," American Journal of Transplantation, vol. 15, 1, pp. 174-251, 2015.

[2] R. W. Busuttil and K. Tanaka, "The utility of marginal donors in liver transplantation," Liver Transplantation, vol. 9, no. 7, pp. 651-663, 2003.

[3] S. Reddy, J. Greenwood, N. Maniakin et al., "Non-heart-beating donor porcine livers: The adverse effect of cooling," Liver Transplantation, vol. 11, no. 1, pp. 35-38, 2005.

[4] D. P. Foley, L. A. Fernandez, G. Leverson et al., "Biliary complications after liver transplantation from donation after cardiac death donors: an analysis of risk factors and long-term outcomes from a single center," Annals of Surgery, vol. 253, no. 4, pp. 817-825, 2011.

[5] J. C. Coffey, K. N. Wanis, D. Monbaliu et al., "The influence of functional warm ischemia time on DCD liver transplant recipients' outcomes," Clinical Transplantation, vol. 31, no. 10, Article ID e13068, 2017.

[6] S. Op Den Dries, M. E. Sutton, N. Karimian et al., "Hypothermic oxygenated machine perfusion prevents arteriolonecrosis of the peribiliary plexus in pig livers donated after circulatory death," PLoS ONE, vol. 9, no. 2, Article ID e88521, 2014.

[7] K. Vekemans, Q. Liu, J. Brassil, M. Komuta, J. Pirenne, and D. Monbaliu, "Influence of Flow and Addition of Oxygen During Porcine Liver Hypothermic Machine Perfusion," Transplantation Proceedings, vol. 39, no. 8, pp. 2647-2651, 2007.

[8] E. Gringeri, P. Bonsignore, D. Bassi et al., "Subnormothermic machine perfusion for non-heart-beating donor liver grafts preservation in a swine model: A new strategy to increase the donor pool?" Transplantation Proceedings, vol. 44, no. 7, pp. 2026-2028, 2012.

[9] J. M. Knaak, V. N. Spetzler, N. Goldaracena et al., "Subnormothermic ex vivo liver perfusion reduces endothelial cell and bile duct injury after donation after cardiac death pig liver transplantation," Liver Transplantation, vol. 20, no. 11, pp. 12961305, 2014.

[10] J. V. Guarrera, S. D. Henry, B. Samstein et al., "Hypothermic machine preservation in human liver transplantation: The first clinical series," American Journal of Transplantation, vol. 10, no. 2, pp. 372-381, 2010.

[11] A. Nassar, Q. Liu, K. Farias et al., "Impact of Temperature on Porcine Liver Machine Perfusion From Donors After Cardiac Death," Artificial Organs, vol. 40, no. 10, pp. 999-1008, 2016.

[12] M. U. Boehnert, J. C. Yeung, F. Bazerbachi et al., "Normothermic acellular ex vivo liver perfusion reduces liver and bile duct injury of pig livers retrieved after cardiac death," American Journal of Transplantation, vol. 13, no. 6, pp. 1441-1449, 2013.

[13] R. B. M. de Vries, K. E. Wever, M. T. Avey, M. L. Stephens, E. S. Sena, and M. Leenaars, "The usefulness of systematic reviews of animal experiments for the design of preclinical and clinical studies," ILAR Journal, vol. 55, no. 3, pp. 427-437, 2014.

[14] C. R. Hooijmans, M. M. Rovers, R. B. M. De Vries, M. Leenaars, M. Ritskes-Hoitinga, and M. W. Langendam, "SYRCLE's risk of 
bias tool for animal studies," BMC Medical Research Methodology, vol. 14, no. 1, article no. 43, 2014.

[15] T. A. Furukawa, C. Barbui, A. Cipriani, P. Brambilla, and N. Watanabe, "Imputing missing standard deviations in metaanalyses can provide accurate results," Journal of Clinical Epidemiology, vol. 59, no. 1, pp. 7-10, 2006.

[16] J. P. T. Higgins, S. G. Thompson, J. J. Deeks, and D. G. Altman, "Measuring inconsistency in meta-analyses," British Medical Journal, vol. 327, no. 7414, pp. 557-560, 2003.

[17] Q. Liu, A. Nassar, K. Farias et al., "Sanguineous normothermic machine perfusion improves hemodynamics and biliary epithelial regeneration in donation after cardiac death porcine livers," Liver Transplantation, vol. 20, no. 8, pp. 987-999, 2014.

[18] B. Banan, H. Chung, Z. Xiao et al., "Normothermic extracorporeal liver perfusion for donation after cardiac death (DCD) livers," Surgery, vol. 158, no. 6, pp. 1642-1650, 2015.

[19] A. Nassar, Q. Liu, K. Farias et al., "Ex vivo normothermic machine perfusion is safe, simple, and reliable: Results from a large animal model," Surgical Innovation, vol. 22, no. 1, pp. 6169, 2015.

[20] Q. Liu, A. Nassar, K. Farias et al., "Comparing Normothermic Machine Perfusion Preservation with Different Perfusates on Porcine Livers from Donors after Circulatory Death," American Journal of Transplantation, vol. 16, no. 3, pp. 794-807, 2016.

[21] S. D. St Peter, C. J. Imber, I. Lopez, D. Hughes, and P. J. Friend, "Extended preservation of non-heart-beating donor livers with normothermic machine perfusion," British Journal of Surgery, vol. 89, no. 5, pp. 609-616, 2002.

[22] M. R. Schön, O. Kollmar, S. Wolf et al., "Liver transplantation after organ preservation with normothermic extracorporeal perfusion," Annals of Surgery, vol. 233, no. 1, pp. 114-123, 2001.

[23] C. Fondevila, A. J. Hessheimer, M.-H. J. Maathuis et al., "Superior preservation of DCD livers with continuous normothermic perfusion," Annals of Surgery, vol. 254, no. 6, pp. 1000-1007, 2011.

[24] M. U. Boehnert, J. Yeung, J.-M. Knaak et al., "Acellular Normothermic Ex Vivo Liver Perfusion (Nevlp) Decreases Hepatocyte and Bile Duct Injury in Livers Retrieved After Cardiac Death," Hepatology, vol. 54, 1, p. 386A, 2011.

[25] M. U. Boehnert, J. C. Yeung, J. M. Knaak, N. Selzner, and M. Selzner, "Normothermic acellular ex vivo liver perfusion (NEVLP) reduces liver and bile duct in DCD liver grafts," American Journal of Transplantation, vol. 13, no. 12, p. 3290, 2013.

[26] T. Vogel, J. G. Brockmann, A. Quaglia et al., "The 24-hour normothermic machine perfusion of discarded human liver grafts," Liver Transplantation, vol. 23, no. 2, pp. 207-220, 2017.

[27] R. Ravikumar, H. Leuvenink, and P. J. Friend, "Normothermic liver preservation: A new paradigm?" Transplant International, vol. 28, no. 6, pp. 690-699, 2015.

[28] T. Shigeta, N. Matsuno, H. Obara et al., "Impact of rewarming preservation by continuous machine perfusion: Improved posttransplant recovery in pigs," Transplantation Proceedings, vol. 45, no. 5, pp. 1684-1689, 2013.

[29] B. Banan, Z. Xiao, R. Watson et al., "Novel strategy to decrease reperfusion injuries and improve function of cold-preserved livers using normothermic ex vivo liver perfusion machine," Liver Transplantation, vol. 22, no. 3, pp. 333-343, 2016.

[30] H. Obara, N. Matsuno, T. Shigeta, T. Hirano, S. Enosawa, and H. Mizunuma, "Temperature controlled machine perfusion system for liver," Transplantation Proceedings, vol. 45, no. 5, pp. 1690-1692, 2013.
[31] T. Minor, P. Efferz, M. Fox, J. Wohlschlaeger, and B. Lüer, "Controlled oxygenated rewarming of cold stored liver grafts by thermally graduated machine perfusion prior to reperfusion," American Journal of Transplantation, vol. 13, no. 6, pp. 14501460, 2013.

[32] R. Bellomo, B. Marino, G. Starkey et al., "Extended normothermic extracorporeal perfusion of isolated human liver after warm ischaemia: a preliminary report," Critical Care And Resuscitation: Journal of The Australasian Academy of Critical Care Medicine, vol. 16, no. 3, pp. 197-201, 2014.

[33] B. Banan, R. Watson, M. Xu, Y. Lin, and W. Chapman, "Development of a normothermic extracorporeal liver perfusion system toward improving viability and function of human extended criteria donor livers," Liver Transplantation, vol. 22, no. 7, pp. 979-993, 2016

[34] S. Op Den Dries, N. Karimian, M. E. Sutton et al., "Ex vivo normothermic machine perfusion and viability testing of discarded human donor livers," American Journal of Transplantation, vol. 13, no. 5, pp. 1327-1335, 2013.

[35] M. E. Sutton, S. Op Den Dries, N. Karimian et al., "Criteria for Viability Assessment of Discarded Human Donor Livers during Ex Vivo Normothermic Machine Perfusion," PLoS ONE, vol. 9, no. 11, Article ID el10642, 2014.

[36] C. J. E. Watson, V. Kosmoliaptsis, L. V. Randle et al., "Normothermic perfusion in the assessment and preservation of declined livers before transplantation: Hyperoxia and vasoplegia-important lessons from the first 12 cases," Transplantation, vol. 101, no. 5, pp. 1084-1098, 2017.

[37] H. Mergental, M. T. P. R. Perera, R. W. Laing et al., "Transplantation of Declined Liver Allografts Following Normothermic ExSitu Evaluation," American Journal of Transplantation, vol. 16, no. 11, pp. 3235-3245, 2016.

[38] R. Ravikumar, W. Jassem, H. Mergental et al., "Liver Transplantation After Ex Vivo Normothermic Machine Preservation: A Phase 1 (First-in-Man) Clinical Trial," American Journal of Transplantation, vol. 16, no. 6, pp. 1779-1787, 2016.

[39] M. Selzner, N. Goldaracena, J. Echeverri et al., "Normothermic ex vivo liver perfusion using steen solution as perfusate for human liver transplantation: First North American results," Liver Transplantation, vol. 22, no. 11, pp. 1501-1508, 2016.

[40] M. Bral, B. Gala-Lopez, D. Bigam et al., "Preliminary SingleCenter Canadian Experience of Human Normothermic Ex Vivo Liver Perfusion: Results of a Clinical Trial," American Journal of Transplantation, vol. 17, no. 4, pp. 1071-1080, 2017. 


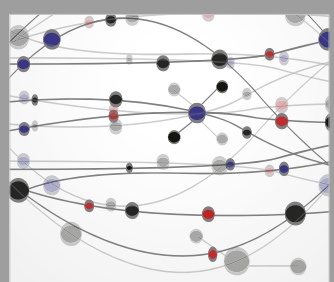

The Scientific World Journal
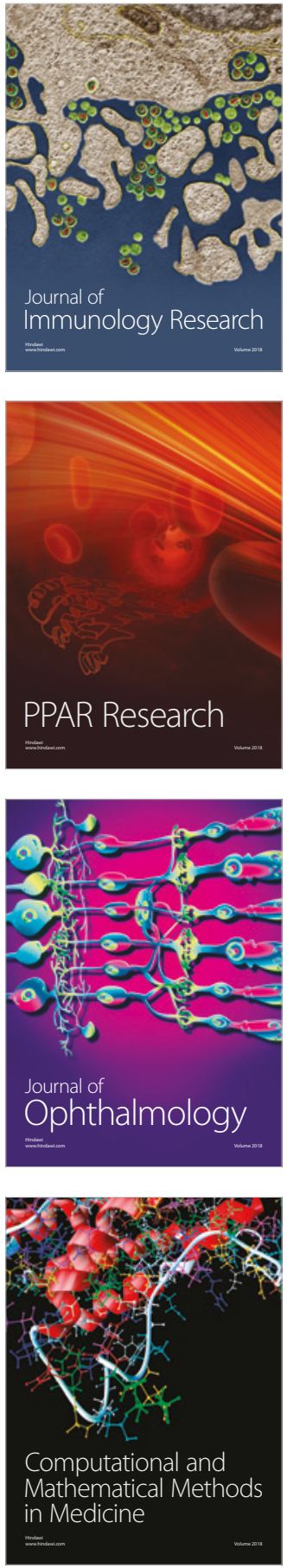

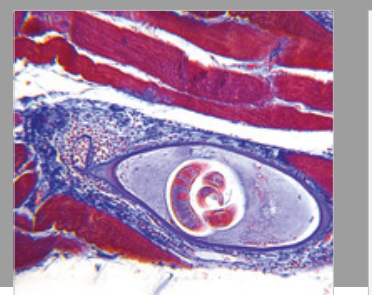

Gastroenterology Research and Practice

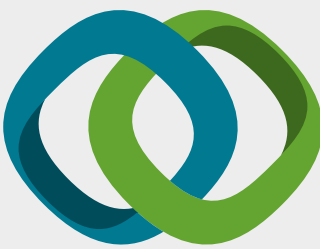

\section{Hindawi}

Submit your manuscripts at

www.hindawi.com
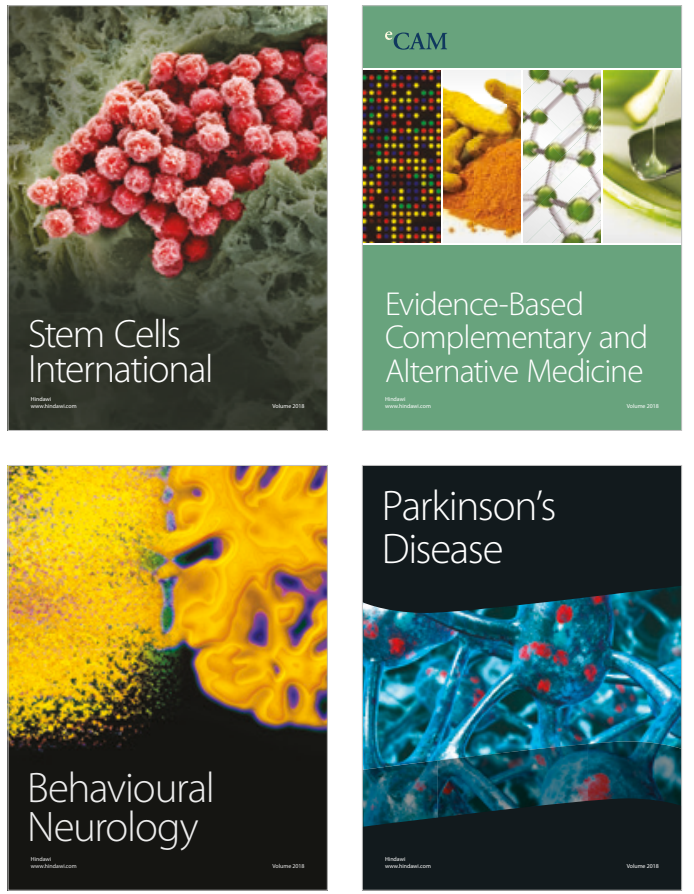

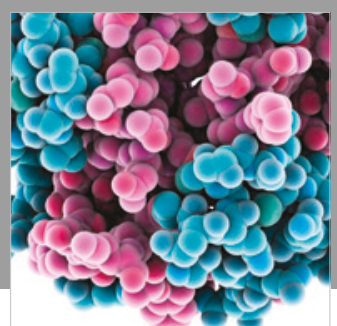

ournal of

Diabetes Research

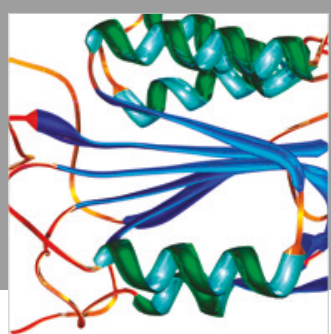

Disease Markers
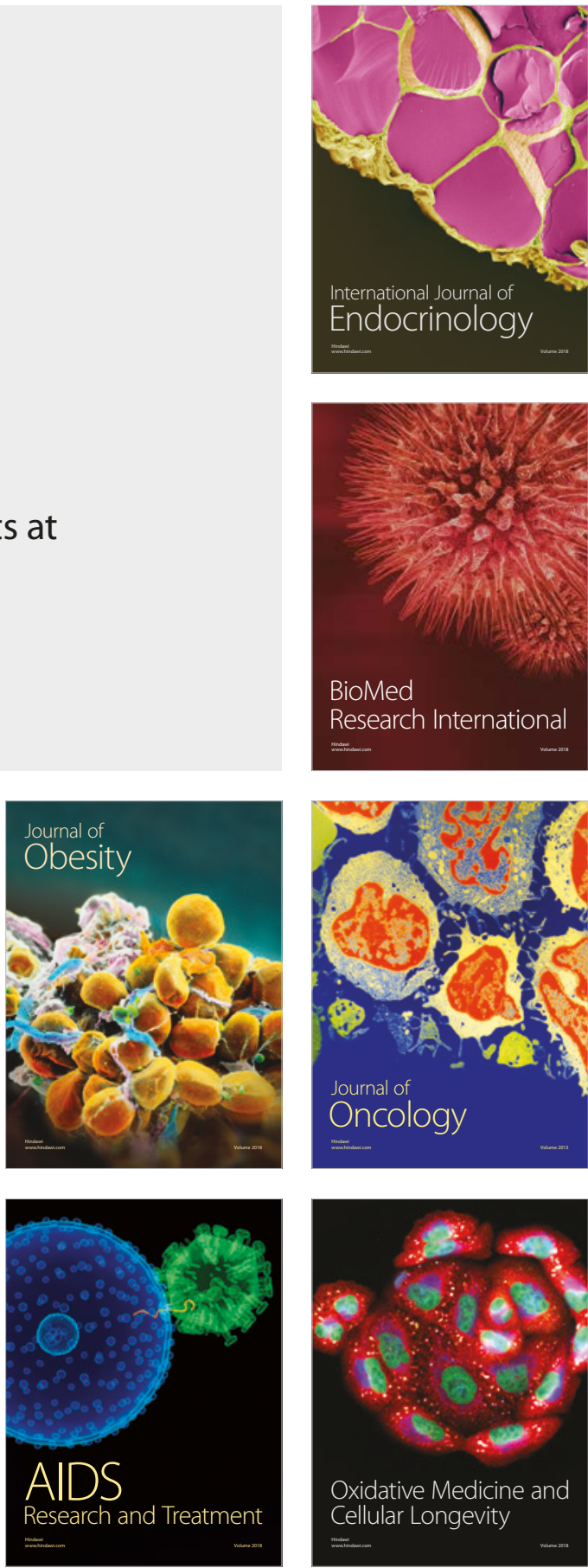